Developing and Piloting a Tool To Create Dot Plots To Summarize Pooled Data for Multiple Outcomes in Systematic Reviews

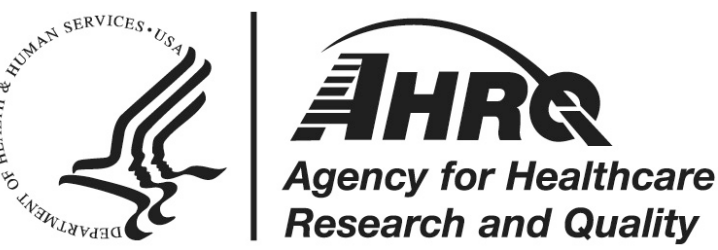




\section{Developing and Piloting a Tool To Create Dot Plots To Summarize Pooled Data for Multiple Outcomes in Systematic Reviews}

Prepared for:

Agency for Healthcare Research and Quality

U.S. Department of Health and Human Services

5600 Fishers Lane

Rockville, MD 20857

www.ahrq.gov

Contract No. 75Q80120D00006

Prepared by:

Pacific Northwest Evidence-based Practice Center

Portland, OR

Investigators:

Yun Yu, M.S.

Rongwei Fu, Ph.D.

Jesse Wagner, M.A.

Azrah Ahmed, B.A.

Connor Smith, M.S.

Roger Chou, M.D.

AHRQ Publication No. 22-EHC001

October 2021 
This report is based on research conducted by the Pacific Northwest Evidence-based Practice Center (EPC) under contract to the Agency for Healthcare Research and Quality (AHRQ), Rockville, MD (Contract No. 75Q80120D00006). The findings and conclusions in this document are those of the authors, who are responsible for its contents; the findings and conclusions do not necessarily represent the views of AHRQ. Therefore, no statement in this report should be construed as an official position of AHRQ or of the U.S. Department of Health and Human Services.

\section{None of the investigators have any affiliations or financial involvement that conflicts with the material presented in this report.}

The information in this report is intended to help healthcare decision makers - patients and clinicians, health system leaders, and policymakers, among others-make well-informed decisions and thereby improve the quality of health care services. This report is not intended to be a substitute for the application of clinical judgment. Anyone who makes decisions concerning the provision of clinical care should consider this report in the same way as any medical reference and in conjunction with all other pertinent information, i.e., in the context of available resources and circumstances presented by individual patients.

This report is made available to the public under the terms of a licensing agreement between the author and the Agency for Healthcare Research and Quality. This report may be used and reprinted without permission except those copyrighted materials that are clearly noted in the report. Further reproduction of those copyrighted materials is prohibited without the express permission of copyright holders.

AHRQ or U.S. Department of Health and Human Services endorsement of any derivative products that may be developed from this report, such as clinical practice guidelines, other quality enhancement tools, or reimbursement or coverage policies may not be stated or implied.

Suggested citation: Yu Y, Fu R, Wagner J, Ahmed A, Smith C, Chou R. Developing and Piloting a Tool To Create Dot Plots To Summarize Pooled Data for Multiple Outcomes in Systematic Reviews. Methods Research Report. (Prepared by the Pacific Northwest Evidencebased Practice Center under Contract No. 75Q80120D00006. AHRQ Publication No. 22EHC001. Rockville, MD: Agency for Healthcare Research and Quality. October 2021. doi: 10.23970/AHRQEPCMETHODSDOTPLOTS.

Posted final reports are located on the Effective Health Care Program 


\section{Preface}

The Agency for Healthcare Research and Quality (AHRQ), through its Evidence-based Practice Centers (EPCs), sponsors the development of evidence reports and technology assessments to assist public- and private-sector organizations in their efforts to improve the quality of health care in the United States. The reports and assessments provide organizations with comprehensive, science-based information on common, costly medical conditions and new health care technologies and strategies. The EPCs systematically review the relevant scientific literature on topics assigned to them by AHRQ and conduct additional analyses when appropriate prior to developing their reports and assessments.

To improve the scientific rigor of these evidence reports, AHRQ supports empiric research by the EPCs to help understand or improve complex methodologic issues in systematic reviews. These methods research projects are intended to contribute to the research base in and be used to improve the science of systematic reviews. They are not intended to be guidance to the EPC program, although may be considered by EPCs along with other scientific research when determining EPC program methods guidance.

AHRQ expects that the EPC evidence reports and technology assessments will inform individual health plans, providers, and purchasers as well as the health care system as a whole by providing important information to help improve health care quality. The reports undergo peer review prior to their release as a final report.

If you have comments on this Methods Research Project they may be sent by mail to the Task Order Officer named below at: Agency for Healthcare Research and Quality, 5600 Fishers Lane, Rockville, MD 20857, or by email to epc@ahrq.hhs.gov.

David Meyers, M.D. Acting Director Agency for Healthcare Research and Quality

Craig A. Umscheid, M.D., M.S.

Director

Evidence-based Practice Center Program

Center for Evidence and Practice Improvement

Agency for Healthcare Research and Quality
Arlene S. Bierman, M.D., M.S.

Director

Center for Evidence and Practice

Improvement

Agency for Healthcare Research and Quality

Christine Chang, M.D., M.P.H.

Task Order Officer

Center for Evidence and Practice

Improvement

Agency for Healthcare Research and Quality 


\section{Acknowledgments}

This work is adopted from the aedots module package. We thank Dr. Rachel Phillips and Dr.

Suzie Cro (Imperial College London, United Kingdom) for their contributions on developing the aedots package. 


\section{Contents}

Introduction ................................................................................................................................................... 1

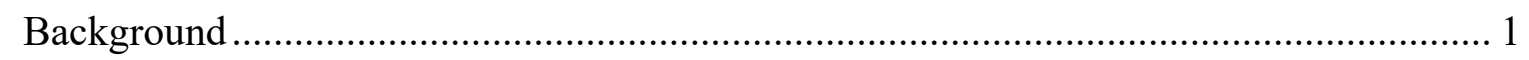

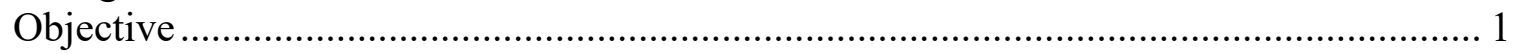

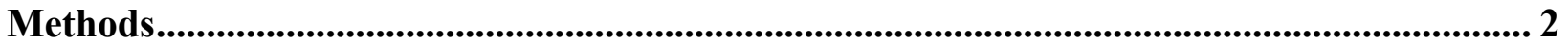

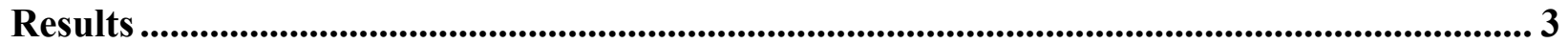

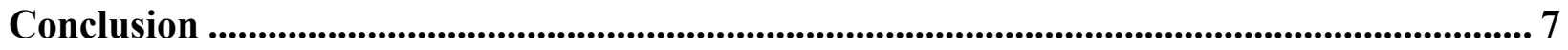

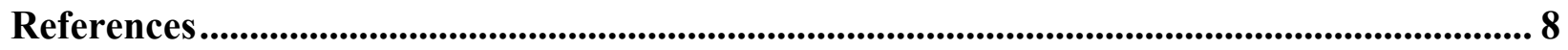

\section{Tables}

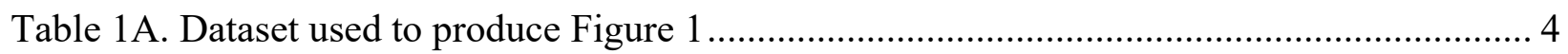

Table 1B. Data dictionary for Table 1A ……………......................................................... 5

Table 2A. Dataset used to produce Figure 2 ………….......................................................... 5

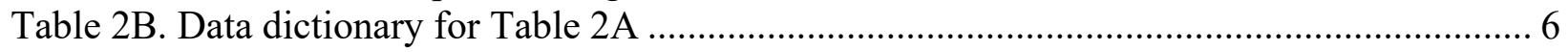

Table 3A. Dataset (0-100 scale) used to produce Figure 3 .......................................................... 6

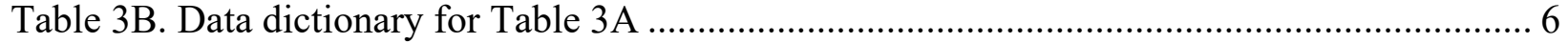

\section{Figures}

Figure 1. Dot plot for dichotomous outcomes, statins versus placebo or no statin ........................ 3

Figure 2. Dot plot for dichotomous outcomes, opioids versus placebo or no opioid ...................... 3

Figure 3. Dot plot for continuous outcomes, opioids versus placebo or no opioid .......................... 4

\section{Appendixes}

Appendix A. Stata Code for Producing Dichotomous Outcomes Shown in Figures 1 and 2 Appendix B. Stata Code for Producing Continuous Outcomes Shown in Figure 3 


\section{Introduction}

\section{Background}

Systematic reviews often assess multiple outcomes (benefits and harm). An ongoing challenge is how to present results for multiple outcomes in a clear and concise way, in order to facilitate judgments of the overall balance of benefits and harms. Dot plots are a data visualization method to display findings across multiple outcomes in a visually pleasing format. In the March 2021 web conference titled "Methods Symposium: Advanced Methods and Innovative Technologies for Evidence Synthesis" funded by the Agency for Healthcare Research and Quality (AHRQ; R13HS027701), focusing on data visualization for adverse events in randomized trials, Dr. Rachel Phillips presented a dot plot as an example data visualization method. ${ }^{1}$ In her example, the dot plot summarized serious adverse events from an individual randomized trial. ${ }^{2}$ The dot plot shows the relative risk with 95 percent confidence intervals as well as the absolute rates in each arm. Having both outcomes in the same plot is useful for interpreting the magnitude of effect. The dot plot also shows the number of adverse events in each group.

A Stata module developed by Phillips and colleagues is available to produce such dot plots (https://ideas.repec.org/c/boc/bocode/s458735.html). ${ }^{3}$ However, the Stata module developed by Phillips and colleagues is intended for display of data from an individual study; it is not designed for use with pooled data. We were unable to locate a module to produce dot plots for pooled data in Stata or another statistical package. In a personal communication, Dr. Phillips stated that she was not aware of such a module being available. In addition, dot plots do not need to be restricted to harms and also could be adapted to summarize findings for continuous as well as dichotomous outcomes. Although commercially available data visualization software can produce plots that display findings for multiple outcomes, this requires purchasing/having the software and uploading the data; in addition, the default plots in currently available data visualization software packages do not display the data (e.g., both the absolute rates and relative risks) in the same format as the dot plots. The availability of a statistical package module to easily produce dot plots would enable Evidence-based Practice Centers (EPC) and other systematic reviewers to more easily include such figures in reports and other products summarizing the findings for multiple outcomes in a single figure, without having to use additional commercial software. This would enhance the usability of EPC and other systematic reviews.

\section{Objective}

The purpose of this methods project was to develop a tool for a standard statistical package (Stata) to create dot plots to summarize pooled data for multiple outcomes in systematic reviews. Our tool could be used for outcomes that are benefits as well as harms. We also attempted to adapt the dot plots to display pooled data for continuous outcomes. 


\section{Methods}

We created a Stata module to produce dot plots from pooled data for multiple outcomes (benefits or harms). For dichotomous outcomes, the dot plots show for each outcome the pooled absolute rate of events in each treatment arm as well as the pooled relative risk and 95 percent confidence interval. The plots also include columns that display the total numerator and denominator for each outcome, the number of trials, the heterogeneity statistic $\left(\mathrm{I}^{2}\right)$, and the strength of evidence grade. For continuous outcomes, the dot plots show for each outcome the baseline pooled weighted mean in the treated and control groups and the pooled mean difference and 95 percent confidence interval, as well as the number of trials, I-square, and strength of evidence grade.

We piloted the dot plot module using pooled data from two completed AHRQ-funded reviews conducted by the Pacific Northwest EPC: a review on statins for primary prevention of cardiovascular events ${ }^{4}$ and a review on opioids for chronic pain. ${ }^{5}$ The statins review focused on dichotomous outcomes; for the pilot we used pooled data for statins versus placebo or no statin and risk of all-cause mortality, cardiovascular mortality, stroke (fatal or nonfatal), myocardial infarction (fatal or nonfatal), revascularization, composite cardiovascular outcomes, withdrawal due to adverse events, cancer, diabetes, myalgia, and liver enzyme abnormalities.

The opioids review reported dichotomous and continuous outcomes; for the pilot we used pooled data for the comparison involving opioids versus placebo or no opioids. For dichotomous outcomes, the pilot utilized pooled data for pain response (the proportion of patients meeting a threshold for improvement in pain), discontinuation due to adverse events, serious adverse events, nausea, vomiting, constipation, somnolence, dizziness, and pruritus. For continuous outcomes, the pilot was restricted to outcomes that used the same scale to report outcomes: pain (mean improvement in pain intensity, transformed from a 0 to 10 scale in the report to a 0 to 100 scale), Short Form (SF)-36 physical function, and SF-36 mental function SF-36 (scored on a 0 to 100 scale).

To create the dot plots, we extracted data from the reviews for the pooled dichotomous and continuous outcomes described above (Tables 1a, 1b, 2a, and 2b). For dichotomous outcomes the dataset used to generate the plots were: outcome, number of trials, numerator and denominator for the treatment and control groups along with the proportion of patients who experienced an outcome in each group, relative risk with upper and lower limit of the confidence interval, Isquare value, and strength of evidence grade. For continuous outcomes (opioid review only), the dataset consisted of: outcome, number of trials, baseline mean value, mean difference with upper and lower limit of the confidence interval, I-square, and strength of evidence (Tables 3a and 3b). The Stata code used to produce the dot plots is shown in Appendix A (dichotomous outcomes) and Appendix B (continuous outcomes).

The Stata modules are being submitted to the Statistical Software Components archive (https://ideas.repec.org/s/boc/bocode.html), a publicly accessible website, for downloading by systematic reviewers who wish to use it. 


\section{Results}

The dot plots can be created using the Stata modules with datasets as shown in Tables 1A, 1B, 2A, 2B, 3A, and 3B. The dot plots are shown in Figures 1, 2, and 3.

\section{Figure 1. Dot plot for dichotomous outcomes, statins versus placebo or no statin}

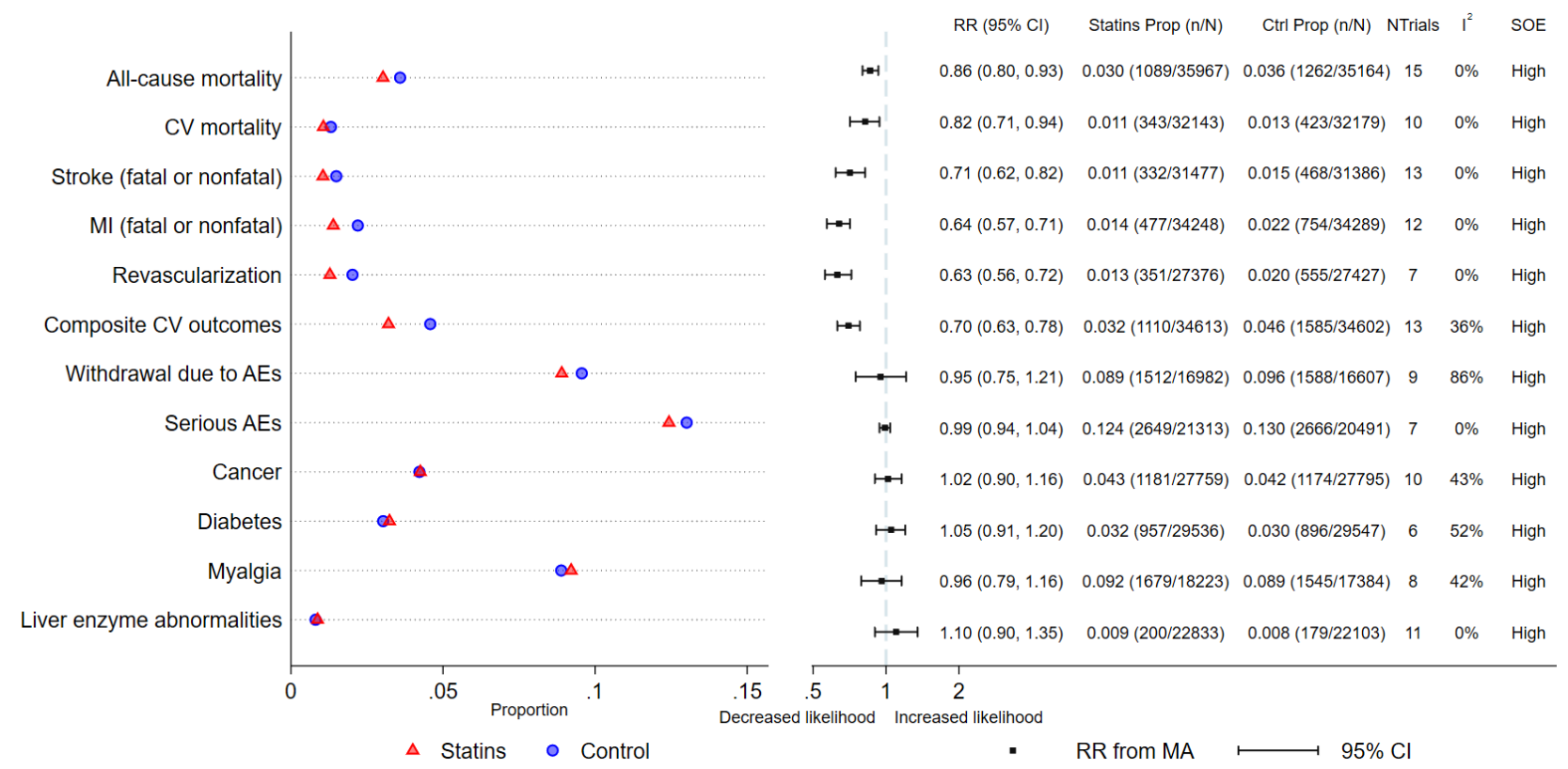

Abbreviations: $\mathrm{AE}=$ adverse event; $\mathrm{ALT}=$ alanine transaminase; $\mathrm{AST}=$ aspartate aminotransferase $\mathrm{CI}=$ confidence interval; $\mathrm{Ctrl}=$ control; $\mathrm{CV}=$ cardiovascular; $\mathrm{MA}=$ meta-analysis; $\mathrm{MI}=$ myocardial infarction; Prop = proportion; $\mathrm{RR}=$ relative risk;

$\mathrm{SOE}=$ strength of evidence

\section{Figure 2. Dot plot for dichotomous outcomes, opioids versus placebo or no opioid}

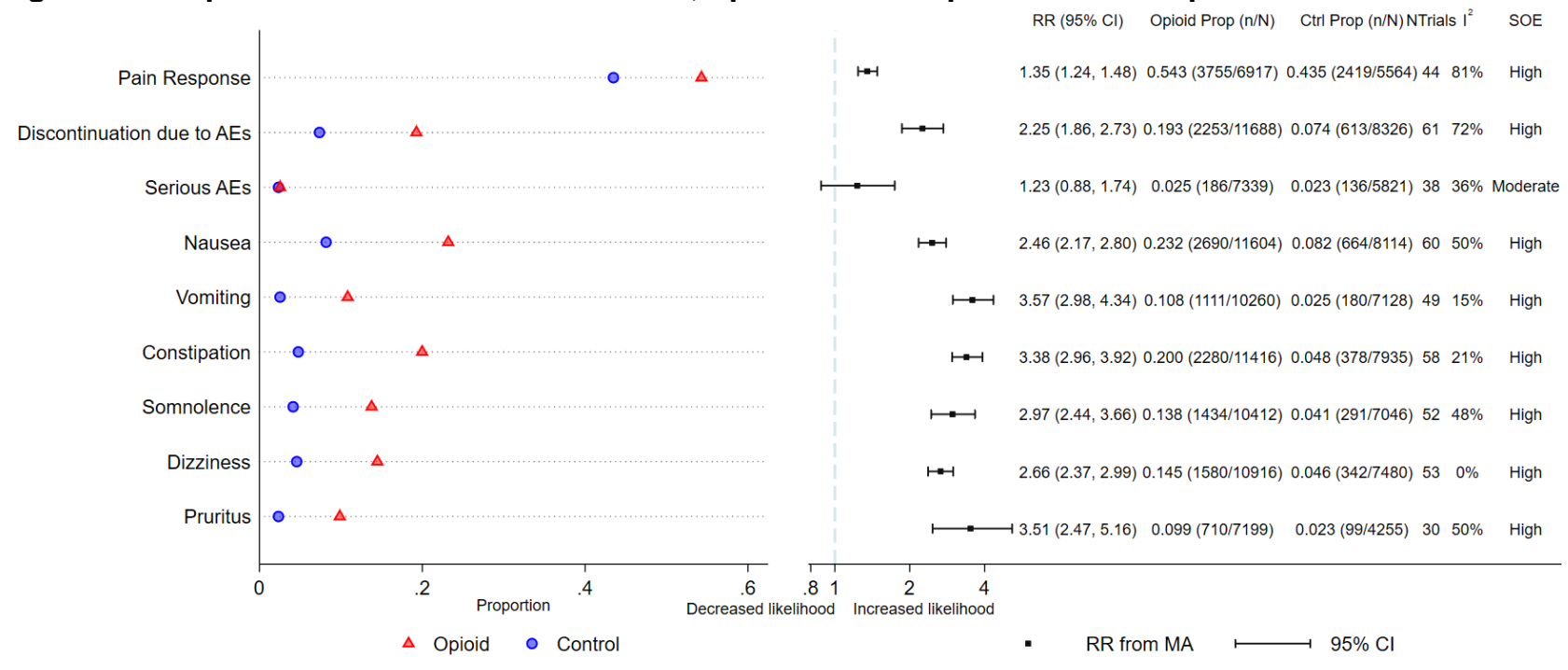

Note: Pain response indicates the proportion of patients who experience $\geq 30 \%$ improvement in pain 
Figure 3. Dot plot for continuous outcomes, opioids versus placebo or no opioid

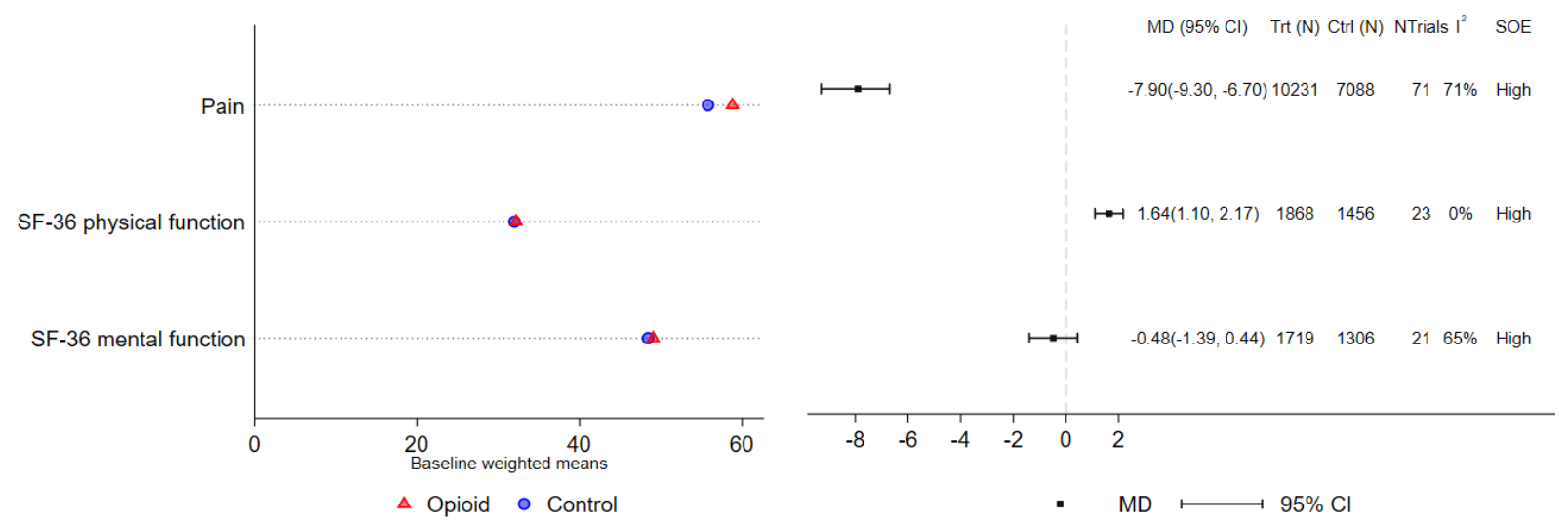

Abbreviations: $\mathrm{CI}=$ confidence interval; $\mathrm{Ctrl}=$ control; $\mathrm{MD}=$ mean difference; $\mathrm{SF}-36=36$ item short form survey; $\mathrm{SOE}=$ strength of evidence; Trt $=$ treatment

Table 1A. Dataset used to produce Figure 1

\begin{tabular}{|c|c|c|c|c|c|c|c|c|c|c|}
\hline Outcome & $\begin{array}{l}\text { No. of } \\
\text { Trials }\end{array}$ & trt_n & trt_N & ctrl_n & ctrl_N & $\mathbf{R} \mathbf{R}$ & cil & ciu & $\begin{array}{c}\text { I- } \\
\text { squared }\end{array}$ & SOE \\
\hline $\begin{array}{l}\text { All-cause } \\
\text { Mortality }\end{array}$ & 15 & 1089 & 35967 & 1262 & 35164 & 0.86 & 0.80 & 0.93 & 0 & High \\
\hline CV Mortality & 10 & 343 & 32143 & 423 & 32179 & 0.82 & 0.71 & 0.94 & 0 & High \\
\hline $\begin{array}{l}\text { Stroke (Fatal or } \\
\text { Nonfatal) }\end{array}$ & 13 & 332 & 31477 & 468 & 31386 & 0.71 & 0.62 & 0.82 & 0 & High \\
\hline $\begin{array}{l}\text { MI (Fatal or } \\
\text { Nonfatal) }\end{array}$ & 12 & 477 & 34248 & 754 & 34289 & 0.64 & 0.57 & 0.71 & 0 & High \\
\hline Revascularization & 7 & 351 & 27376 & 555 & 27427 & 0.63 & 0.56 & 0.72 & 0 & High \\
\hline $\begin{array}{l}\text { Composite CV } \\
\text { Outcomes }\end{array}$ & 13 & 1110 & 34613 & 1585 & 34602 & 0.70 & 0.63 & 0.78 & 0.36 & High \\
\hline $\begin{array}{l}\text { Withdrawal Due } \\
\text { to AEs }\end{array}$ & 9 & 1512 & 16982 & 1588 & 16607 & 0.95 & 0.75 & 1.21 & 0.86 & High \\
\hline Serious AEs & 7 & 2649 & 21313 & 2666 & 20491 & 0.99 & 0.94 & 1.04 & 0 & High \\
\hline Cancer & 10 & 1181 & 27759 & 1174 & 27795 & 1.02 & 0.90 & 1.16 & 0.43 & High \\
\hline Diabetes & 6 & 957 & 29536 & 896 & 29547 & 1.05 & 0.91 & 1.20 & 0.52 & High \\
\hline Myalgia & 8 & 1679 & 18223 & 1545 & 17384 & 0.96 & 0.79 & 1.16 & 0.42 & High \\
\hline $\begin{array}{l}\text { Liver Enzyme } \\
\text { Abnormalities }\end{array}$ & 11 & 200 & 22833 & 179 & 22103 & 1.10 & 0.90 & 1.35 & 0 & High \\
\hline
\end{tabular}

Abbreviations: $\mathrm{AE}=$ adverse event; $\mathrm{ALT}=$ alanine aminotransferase; $\mathrm{AST}=$ aspartate aminotransferase; $\mathrm{CV}=$ cardiovascular; $\mathrm{MI}=$ myocardial infarction 
Table 1B. Data dictionary for Table 1A

\begin{tabular}{|l|l|}
\hline Variable Name & Description \\
\hline Outcome & Outcome name \\
\hline No. of Trials & Number of trials \\
\hline trt_n & Number of events of treatment group \\
\hline trt_N & Total N of treatment group \\
\hline ctrl_n & Number of events in control group \\
\hline ctrl_N & Total N of control group \\
\hline RR & Pooled risk ratio from meta-analysis \\
\hline Cil & lower bound of $95 \%$ Cl of RR \\
\hline Ciu & upper bound of $95 \%$ Cl of RR \\
\hline I-squared & l-square for the RR \\
\hline SOE & Strength of evidence \\
\hline Abbreviations: CI $=$ & confidence interval
\end{tabular}

Abbreviations: $\mathrm{CI}=$ confidence interval

Table 2A. Dataset used to produce Figure 2

\begin{tabular}{|l|c|c|c|c|c|c|c|c|c|c|}
\hline Outcome & $\begin{array}{c}\text { No. of } \\
\text { Trials }\end{array}$ & $\mathbf{t r t} \mathbf{n}$ & $\mathbf{t r t} \mathbf{N}$ & $\mathbf{c t r l} \mathbf{n}$ & $\mathbf{c t r l} \mathbf{N}$ & $\mathbf{R R}$ & $\mathbf{c i l}$ & ciu & $\begin{array}{c}\text { I- } \\
\text { squared }\end{array}$ & SOE \\
\hline Pain Response & 44 & 3755 & 6917 & 2419 & 5564 & 1.35 & 1.24 & 1.48 & 0.81 & High \\
\hline $\begin{array}{l}\text { Discontinuation } \\
\text { Due to AEs }\end{array}$ & 61 & 2253 & 11688 & 613 & 8326 & 2.25 & 1.86 & 2.73 & 0.72 & High \\
\hline Serious AEs & 38 & 186 & 7339 & 136 & 5821 & 1.23 & 0.88 & 1.74 & 0.36 & Moderate \\
\hline Nausea & 60 & 2690 & 11604 & 664 & 8114 & 2.46 & 2.17 & 2.8 & 0.5 & High \\
\hline Vomiting & 49 & 1111 & 10260 & 180 & 7128 & 3.57 & 2.98 & 4.34 & 0.15 & High \\
\hline Constipation & 58 & 2280 & 11416 & 378 & 7935 & 3.38 & 2.96 & 3.92 & 0.21 & High \\
\hline Somnolence & 52 & 1434 & 10412 & 291 & 7046 & 2.97 & 2.44 & 3.66 & 0.48 & High \\
\hline Dizziness & 53 & 1580 & 10916 & 342 & 7480 & 2.66 & 2.37 & 2.99 & 0 & High \\
\hline Pruritus & 30 & 710 & 7199 & 99 & 4255 & 3.51 & 2.47 & 5.16 & 0.5 & High \\
\hline
\end{tabular}

Abbreviations: $\mathrm{AE}=$ adverse event

Note: For variable abbreviations, see Table 2A 
Table 2B. Data dictionary for Table 2A

\begin{tabular}{|l|l|}
\hline Variable Name & Description \\
\hline Outcome & Outcome name \\
\hline No. of Trials & Number of trials \\
\hline trt_n & Number of events of treatment group \\
\hline trt_N & Total N of treatment group \\
\hline ctrl_n & Number of events in control group \\
\hline ctrl_N & Total N of control group \\
\hline RR & Pooled risk ratio from meta-analysis \\
\hline Cil & lower bound of $95 \%$ Cl of RR \\
\hline Ciu & upper bound of $95 \%$ Cl of RR \\
\hline I-squared & l-square for the RR \\
\hline SOE & Strength of evidence \\
\hline
\end{tabular}

Abbreviations: $\mathrm{CI}=$ confidence interval

Table 3A. Dataset (0-100 scale) used to produce Figure 3

\begin{tabular}{|l|c|c|c|c|c|c|c|c|c|c|}
\hline Outcome & $\begin{array}{c}\text { No. of } \\
\text { Trials }\end{array}$ & trt_bl_mean & ctrl_bl_mean & trt_N & ctrl_N & MD & cil & ciu & $\begin{array}{c}\text { I- } \\
\text { squared }\end{array}$ & SOE \\
\hline Pain & 71 & 58.8 & 55.8 & 10231 & 7088 & -7.9 & -9.3 & -6.7 & 0.71 & High \\
\hline $\begin{array}{l}\text { SF-36 } \\
\begin{array}{l}\text { Physical } \\
\text { Function }\end{array}\end{array}$ & 23 & 32.22 & 32.01 & 1868 & 1456 & 1.64 & 1.1 & 2.17 & 0 & High \\
\hline $\begin{array}{l}\text { SF-36 Mental } \\
\text { Function }\end{array}$ & 21 & 49.07 & 48.43 & 1719 & 1306 & -0.48 & -1.39 & 0.44 & 0.65 & High \\
\hline
\end{tabular}

Abbreviations: $\mathrm{SF}=$ Short Form

Note: For variable abbreviations, see Table 3A

Table 3B. Data dictionary for Table 3A

\begin{tabular}{|l|l|}
\hline Variable Name & Description \\
\hline Outcome & Outcome name \\
\hline No. of Trials & Number of trials \\
\hline trt_bl_mean & Weighted mean at baseline of treatment group \\
\hline ctrl_bl_mean & Weighted mean at baseline of treatment group \\
\hline trt_N & Total N of treatment group \\
\hline ctrl_N & Total N of control group \\
\hline MD & Pooled mean difference from meta-analysis \\
\hline Cil & lower bound of $95 \%$ Cl of MD \\
\hline Ciu & upper bound of 95\% Cl of MD \\
\hline I-squared & I-square for the mean difference \\
\hline SOE & Strength of evidence \\
\hline
\end{tabular}

Abbreviations: $\mathrm{CI}=$ confidence interval 


\section{Conclusion}

We created Stata modules to enable systematic reviews to produce dot plots summarizing pooled data for multiple outcomes (either beneficial or harmful). The datasets used to create the dot plots as well as the Stata code are included in this report. Of note, the "trials," "I-squared," and "SOE" columns could be customized to display alternative information. The modules are being uploaded to the Statistical Software Components website, to be publicly accessible for downloading and use.

This was a pilot project and future work could be performed to further develop or refine the Stata modules and dot plots. For example, it may be possible to automate the creation of dot plots by taking data directly from Stata analysis output, eliminating an extra step of entering the required data into a separate table. For continuous outcomes, the issue of outcomes using different scales created an unanticipated challenge and limited the continuous outcomes that could be displayed in a single plot. Future work could explore methods to display results using standardized outcomes (e.g., standardized mean difference [SMD]); however, it is not clear what would be displayed on the left side of the plot, as SMD is a unitless measure without associated baseline values. In addition, because the SMD is a unitless measure, it is difficult for readers to interpret. 


\section{References}

1. Cornelius V, Cro S, Phillips R. Advantages of visualisations to evaluate and communicate adverse event information in randomised controlled trials. Trials. 2020 Dec 22;21(1):1028. doi: 10.1186/s13063020-04903-0. PMID: 33353566.

2. Cornelius V, Cro S, Phillips R. Advantages of visualisations to evaluate and communicate adverse event information in randomised controlled trials [Internet].

Springer Nature; 2020.

https://springernature.figshare.com/collectio ns/Advantages of visualisations to evaluat $\mathrm{e}$ and communicate adverse event inform ation in randomised controlled trials $/ 5248$ 761/1. Accessed Sep 12021.

3. Amit O, Heiberger RM, Lane PW. Graphical approaches to the analysis of safety data from clinical trials. Pharm Stat. 2008 Jan-Mar;7(1):20-35. doi:

10.1002/pst.254. PMID: 17323410.
4. Chou R, Dana T, Blazina I, et al. Statins for Prevention of Cardiovascular Disease in Adults: Evidence Report and Systematic Review for the US Preventive Services Task Force. Jama. 2016 Nov 15;316(19):2008-24. doi: 10.1001/jama.2015.15629. PMID: 27838722 .

5. Chou R, Hartung D, Turner J, et al. AHRQ Comparative Effectiveness Reviews. Opioid Treatments for Chronic Pain. Rockville (MD): Agency for Healthcare Research and Quality (US); 2020. 


\section{Appendix A. Stata Code for Producing Dichotomous Outcomes Shown in Figures 1 and 2}

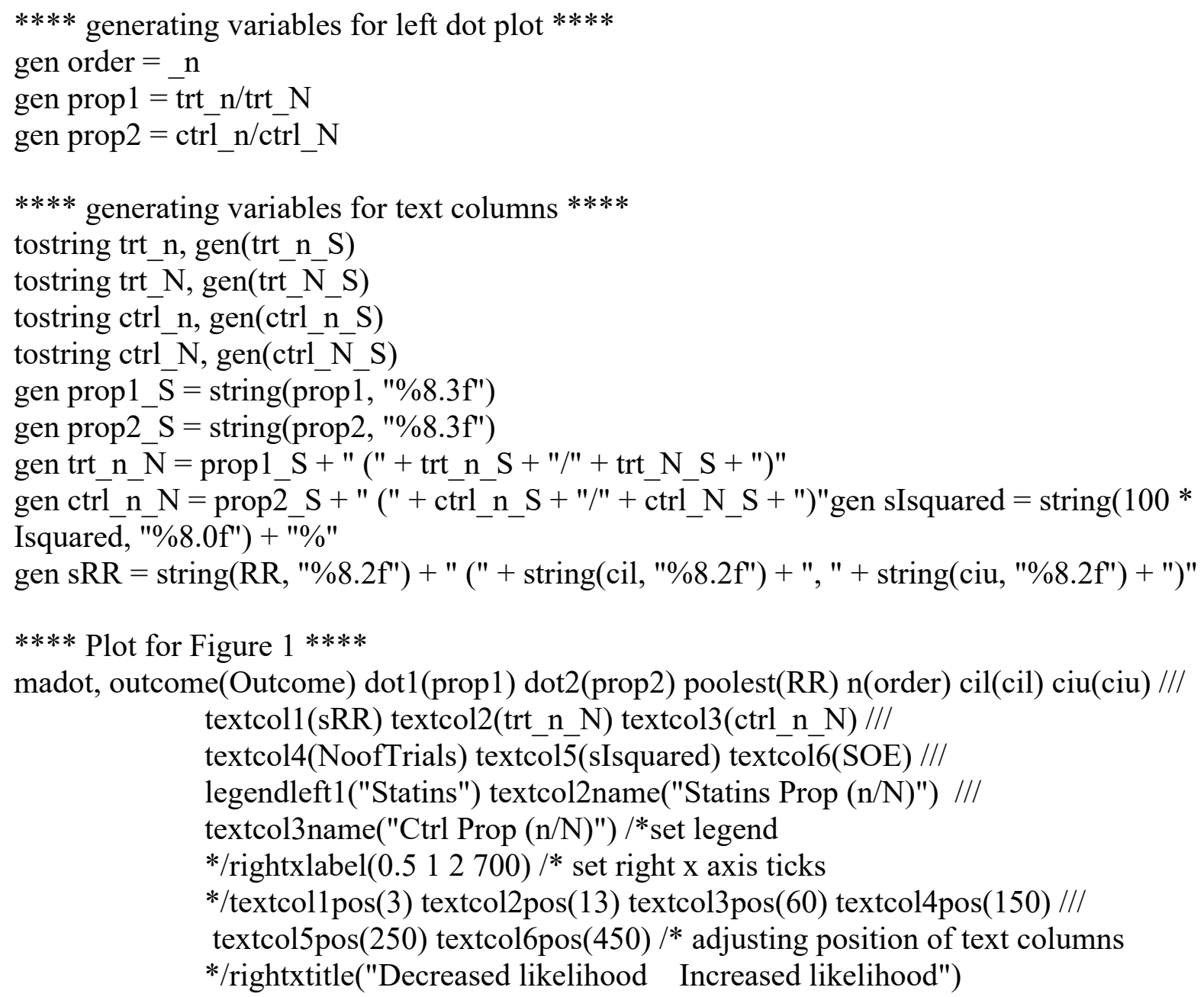

/* Import data from table $2 \mathrm{~A}$ and repeat the steps of generating variables */

madot, outcome(Outcome) dot1(prop1) dot2(prop2) poolest(RR) n(order) cil(cil) ciu(ciu) ///

textcol1(sRR) textcol2(trt_n_N) textcol3(ctrl_n_N) ///

textcol4(NoofTrials) textcol$\overline{5}$ (sIsquared) textcol6(SOE) ///

legendleft1("Opioid") textcol2name("Opioid Prop (n/N)") ///

textcol3name("Ctrl Prop (n/N)") /*set legend

*/rightxlabel(0.8 124 1100)/* set right $\mathrm{x}$ axis ticks

*/textcol1 pos(10) textcol2pos(33) textcol3pos(110) ///

textcol4pos(250) textcol5pos(350) textcol6pos(600)/* adjusting position of text columns

*/graphheight(4.5) graphwidth(10.5)/*set graph height and width

*/rightxtitle("Decreased likelihood Increased likelihood") 


\section{Appendix B. Stata Code for Producing Continuous Outcomes Shown in Figure 3}

gen order $=\_n$

*** generating variables for text columns $* * * *$ gen sIsquared = string $(100 *$ Isquared, "\%8.0f" $)+" \% "$ gen sMD = string $(\mathrm{MD}$, "\%8.2f") + " (" + string(cil, "\%8.2f") + ", " + string(ciu, "\%8.2f") + ")"

*** plot $* * * *$

madot, outcome(Outcome) dot1(trt_bl_mean) dot2(ctrl_bl_mean) poolest(MD) n(order) cil(cil) $\operatorname{ciu}(\mathrm{ciu}) / / /$

textcol1(sMD) textcol2(trt_N) textcol3(ctrl_N) ///

textcol4(NoofTrials) textcol5(sIsquared) textcol6(SOE) ///

$\operatorname{logoff}(1)$ textcolposy $(0.5) / *$ turn off log-cale of $\mathrm{x}$ axis of right plot

*/legendleft1("Opioid") legendleft2("Control")/*set legend

*/textcol2name("Trt (N)") textcol3name("Ctrl (N)")/*set text column names

*/rightxlabel ( -8 - $-6-4-4 \quad-20218) / *$ set right $\mathrm{x}$ axis ticks

*/textcol1pos(5) textcol2pos(8.7) textcol3pos(11) textcol4pos(13.5)

textcol5pos(15) textcol6pos(17)/* adjusting position of text columns

size */

*/graphheight(3) graphwidth(8.5) iscale(0.8)/*set graph height, width and text 